\title{
Topological geometry analysis for complex dynamic systems based on adaptive control method
}

\author{
M. Lellis Thivagar ${ }^{1}$, Abdulsattar Abdullah Hamad ${ }^{2}$ \\ ${ }^{1,2}$ School of Mathematics, Madurai Kamaraj University, Madurai, Tamil Nadu, India
}

\section{Article Info}

Received, June 2019

\section{Keyword:}

Lu dynamic system model, Hamiltonian, synchronization, Lyapunov expansion, stability

\begin{abstract}
Several models had been proposed for dynamic systems, and different criteria had been analyzed for such models such as, Hamiltonian, synchronization, Lyapunov expansion, and stability. The geometry criteria play a significant part in analyzing dynamic systems and some study articles analyze the geometry of such topics. The synchronization and the complex-network control with specified topology, meanwhile, the exact topology may be unknown. In the present paper, and by making use of the adaptive control method, a proposed control method is developed to determine the actual topology. The basic idea in the proposed method is to receive evolution of the network-nodes.
\end{abstract}

\section{Corresponding Author:}

Abdulsattar Abdullah Hamad,

${ }^{2}$ School of Mathematics, Madurai Kamaraj University, Madurai, Tamil Nadu, India

Email: satar198700@gmail.com, al_kasrage@yahoo.com

\section{Introduction}

Since the 1950s and 1960s, new sciences have begun to emerge and capture the curiosity and interest of scientists. Among them are Systems Theory, Chaos Theory, Cybernetics and Artificial Intelligence. Systems theory was founded by the German biologist Ludwig von Bertalanffy, the English economist Kenneth Boulding and others between 1940 and 1970, and is based on the principles of physics, biology and applied engineering. This science then continued its growth and intertwined with many other sciences including: philosophy, sociology, organizational theory, management, economics and other sciences. Systems theory seeks not only to present the world as systems and knowledge of how these systems work and the common links between all existing systems in the world. Are all theoretical, applied and human sciences. Therefore, knowledge and understanding of the principles of systems theory can gain one comprehensive understanding of science and then the whole world that science seeks to read and discover [1]. The whole world is mobile systems or living machines that exist and continue in a certain medium and interact with other systems (machines) and have decay factors inside them and decay factors outside them remain resistant until the collapse of resistance in the end, decomposing its elements to join other systems still work. It applies to everything from galaxies, stars, planets and not to humans and other living things. Systems in the world can be divided into open systems, closed systems (or) simple systems, complex systems (or) fixed systems and mobile systems [2].

\subsection{Open System}

A system that is defined by a basic connection between it and its surroundings. A closed system is a system that tends to be confined to itself and avoids interaction with environmental data, needs, expectations and aspirations. A closed system tends to ignore external considerations. Closed systems are defined by being caught within boundaries that restrict their autonomy and communication with the setting as the structures very nature does not allow it to separate from that system factors from the surroundings. 


\section{2}

\section{Simple system}

It is a system of few elements and few relations between the elements and for this facilitates the study of simple systems on complex systems, and therefore the researcher in any scientific subject to dismantle the complex system into simple systems in order to facilitate the study and understanding of simple systems separately Then study the relationships between these simple systems to know and understand the overall picture of the whole complex system [3]. Complex system: is a system of many elements of many relationships are characterized by complexity, but the difficulty of defining complexity makes the description of complex systems apply to many physical systems such as natural networks or abstract or artificial. The study of these complex systems falls within the so-called (Complexity Sciences), which are interdisciplinary academic disciplines. Examples of complex systems include the social system of ants, the social system of humans, the economy, the nervous system, living cells and the whole organism. Recently, new energy or telecommunications infrastructure has been added, and what is shaped by the modern telecommunications revolution such as the network community. The most important characteristic of these systems is that they share networks in one way or another, their interconnection is complex or not easy to represent [4]. They may contain different and reciprocal components. But each complex system has its own characteristics and even its organized relations between its components. The common property is the difficulty or limited mathematical modeling of these systems. Fixed system: is the system that does not happen to any change over time, its elements are unchanged and relationship between its elements is unchanged. This system cannot exist in nature because all natural systems are dynamic, but a fixed system is a theoretical idea that has been invented to understand and assimilate natural dynamic systems by theoretically breaking them into fixed systems (such as still images we take for ourselves). Find the most stable elements and relationships in natural systems [5]. Examples of fixed systems are: geometric shapes, natural numbers, and mathematical equations describing fixed sentences. Dynamic system: A system that defines sentences regulated by linear differential equations, partial linear differential equations, non-linear differential equations, partial nonlinear differential equations, or algebraic differential equations. Examples of kinetic sentences are all mathematical models describing the movement of a simple pendulum or the flow of water in a pipe and others. For each kinetics states, these states are also the coordinates of the geometrical or state space. Each kinematics rule of evolution is a rule (mathematical function) that describes the correlation of the state of the sentence with time or place and thus determines the future states of the sentence depending on its current state. The rule of evolution of these sentences can be inevitable: for a given period of time, the current state will evolve into another single, pre-determined state of the sentence's evolution function. It can also be a possibility [6]. A dynamic system is a moving machine, and in this sense it can be launched as a description of all the elements and assets in the world. A single atom of any substance is a kinetic machine, every living organism is a kinetic machine, the planet is a kinetic machine, the solar system is a kinetic machine, and all elements of the universe and the world are interdependent machines, machines or dynamic systems that work on each other or in parallel, and dynamic systems or motor machines are not only in kind, there are other motor machines are implicit indirect systems, such as: political, cultural, social, psychological, mental, cognitive and emotional systems that control the lives of human beings, for example. In an orderly conception of human life as a large machine that works and moves as a gear in a larger machine and so on. Let us start defining its meaning from mathematical point of view, the dynamical system from mathematical point of view is that system which has a function that describes the dependency of the time of a point in a geometrical space. The dynamic system distribution rule is a feature that defines what potential states are derived from the present state. [7]. If one turn to physics, the dynamic system could then be defined as a particle whose condition differs over moment and therefore obeys differential equations with time derivatives [8]. The research of dynamic systems is the center of the theory of dynamic systems with a broad spectrum of apps. [9]. The dynamic system may or may not be linear, the scope of the dynamic system [10]. Nonlinear continuous dynamical systems, have been extensively studied to this day. The first trials for such model is the Lu model [11], this type had a significant stake in their phase-space behaviour. Chen [12] had presented a simple 3-D auto system, which is not diffeo-morphic with Lorenz attractor. In the arrangement of values for a parameter k, Lü [13] proposed another 3-D attractor showing chaotic behavior in distinct respects and not diffeo-morphic with Lorenz [14]. In 1963, the first chaotic nonlinear system was suggested by Lorenz [15]. The first trial for establishing a chaotic nonlinear system was proposed by Fowler in 1982, in which was as a generalization of the Lorenz system. The messy structure of the Lorenz system is utilized to suppose the physics of the liquid flows calorific convection [16]. The literature [17] has suggested numerous chaotic and super chaotic complex systems with quadratic nonlinear conditions. These systems are combined with quadratic nonlinear conditions in the same category as the Lorenz equation. In the proposed model, the variables, $\mathrm{x}$, and $\mathrm{y}$ are assumed to be functions for one real and three complex parameters, while, the $\mathrm{z}$ variable is assumed in terms of only real variable only. After long mathematical manipulations, a system of nine equations are obtained which represents the hyper-complex chaotic system. The dynamics of the obtained system were analyzed, which include phase spaces, calculations of eigenvalues and Lyapunov exponents, and all related analyses. The results due to the proposed system gave an acceptable accuracy compared with the corresponding due to 6-D models. A wide range of research papers tried to investigate the geometry nature, complex-dynamic network synchronization and regulate with certain topologies. [18-25]. The true topology of a complex-dynamic network is unknown or at least uncertain in most apps. [26-33]. the network topology recently took more attention in different disciplines, such as the protein-DNA (deoxyribonucleic-acid) interactions in cellular processes. Several models had been proposed for dynamic systems, and different criteria had 
been analyzed for such models such as, Hamiltonian, synchronization, Lyapunov expansion, and stability. The geometry criteria play a significant part in analyzing dynamic systems and some study articles have evaluated such subject geometry. The synchronization and the complex-network control with specified topology, meanwhile, the exact topology may be unknown. In the present paper, and by making use of the adaptive control method, a proposed control method is developed to determine the actual topology. The basic idea in the proposed method is to receive evolution of the network-nodes.

\section{A nine-DECLM dynamic model}

In 2019, a previous paper [33] for the same authors, they suggested and developed a new 9-DECLM mathematical dynamical complex model. In that paper, they established a new mathematical model for a chaotic dynamical system, which leaded to nine equation. The model was characterized by the three following first order ordinary equations:

$$
\begin{gathered}
\frac{d x(t)}{d t}=\alpha(y-x) \\
\frac{d y(t)}{d t}=\gamma x-y-x z \\
\frac{d z(t)}{d t}=-\beta z+\frac{1}{2}(y \bar{x}+x \bar{y})
\end{gathered}
$$

Where

In equations (1-3), $\alpha, \beta, \gamma$ are real parameters and the variables $x, y, z$ are defined as:

$$
\begin{gathered}
x=u_{1}+i u_{2}+j u_{3}+k u_{4} \\
y=u_{5}+i u_{6}+j u_{7}+k u_{8} \\
z=u_{9}
\end{gathered}
$$

The suggested model is achieved by substituting complex variables for real variables of the classical Lu model, this can be seen in equations (4) and (5). The system obtained by the suggested model is highly dimensional. There are two different methods to construct higher dimensional model, the first one can be occurred by adding new variables to the original system. The second one by combining two existing models, this method need a special care to ensure stable system. The first method to construct the existing method was chosen in this paper.

\section{Hamiltonian Dynamics of the 9-DECLM}

The generalized Hamiltonian canonical form takes for the new system [33-36] took the following form:

$$
\dot{x}(x)=\frac{1}{2 \alpha}\left[U_{1}^{2}+U_{2}^{2}+U_{3}^{2}+U_{4}^{2}+U_{5}^{2}+U_{6}^{2}+U_{7}^{2}+U_{8}^{2}+U_{9}^{2}\right]
$$

Equation (7) is a new Hamiltonian form for the suggested new model.

\section{Invariance and symmetry}

The invariance means that both:

And

$$
u_{1}, u_{2}, u_{3}, u_{4}, u_{5}, u_{6}, u_{7}, u_{8}, u_{9}
$$

$$
-u_{1},-u_{2},-u_{3},-u_{4},-u_{5},-u_{6},-u_{7},-u_{8},-u_{9}
$$

Two solutions for the system, and this can be clearly approved by introducing the following substitution:

$$
\begin{aligned}
& u_{K}^{\prime r}=u_{K}^{\prime r} \cos \Theta-u_{K}^{\prime i} \sin \Theta \\
& u_{K}^{\prime i}=u_{K}^{\prime i} \sin \Theta+u_{K}^{\prime i} \cos \Theta \\
& r \text { : real } \\
& i: \text { Imaginary } \\
& \mathrm{K}=1,2,3, \ldots, 9
\end{aligned}
$$

\subsection{Dissipation of the system}

The Dissipation of the system will be examined through the divergence condition as follows:

$$
\nabla \mathrm{v}=\sum_{\mathrm{i}=1}^{\mathrm{n}} \frac{\partial \dot{u}_{i}}{\partial u_{i}}<0
$$

Equation (25) in some details can be written as: 


$$
\nabla v=\frac{\dot{u} u_{1}}{\partial u_{1}}+\frac{\dot{\partial} u_{2}}{\partial u_{2}}+\frac{\dot{\partial u_{3}}}{\partial u_{3}}+\frac{\dot{\partial u_{4}}}{\partial u_{4}}+\frac{\dot{\partial u_{5}}}{\partial u_{5}}+\frac{\dot{\partial} u_{6}}{\partial u_{6}}+\frac{\partial u_{7}}{\partial u_{7}}+\frac{\dot{\partial} u_{8}}{\partial u_{8}}+\frac{\dot{\partial u_{9}}}{\partial u_{9}}
$$

By computing the partial derivatives that appeared in equation (26), and simplifying, leads to the Dissipation condition as follows:

$$
3 \alpha+\beta+3>0
$$

This condition is the basis of the chaotic behavior occurrence.

\subsection{Equilibria}

To study the equilibria, let us find the homogenous solution for the system given by:

$$
\begin{aligned}
& \alpha\left(u_{5}-u_{1}\right)=0 \Rightarrow u_{1}=u_{5} \\
& \alpha\left(u_{6}-u_{2}\right)=0 \Rightarrow u_{2}=u_{6} \\
& \alpha\left(u_{7}-u_{3}\right)=0 \Rightarrow u_{3}=u_{7} \\
& \alpha\left(u_{8}-u_{4}\right)=0 \Rightarrow u_{4}=u_{8} \\
& u_{1}-u_{5}-u_{1} u_{9}=0 \Rightarrow u_{1}\left(\gamma-u_{9}-1\right)=0 \\
& u_{2}-u_{6}-u_{2} u_{9}=0 \Rightarrow u_{2}\left(\gamma-u_{9}-1\right)=0 \\
& u_{3}-u_{7}-u_{3} u_{9}=0 \Rightarrow u_{3}\left(\gamma-u_{9}-1\right)=0 \\
& u_{4}-u_{8}-u_{4} u_{9}=0 \Rightarrow u_{4}\left(\gamma-u_{9}-1\right)=0 \\
& -\beta u_{9}+\left(u_{1}^{2}+u_{2}^{2}+u_{3}^{2}+u_{4}^{2}\right)=0
\end{aligned}
$$

From $5^{\text {th }}$ row in equation (14) till the $8^{\text {th }}$ row, one can obtain the only possible solution that is:

$$
u_{9}=\gamma-1
$$

While the all remaining variables will equal zero solution, i.e.

$$
u_{1}=u_{2}=u_{3}=u_{4}=u_{5}=u_{6}=u_{7}=u_{8}=0
$$

\subsection{Stability}

To study the stability for the proposed system, let us refer back to the Jacobin given by equation (14), and let us find the characteristic equation as follows:

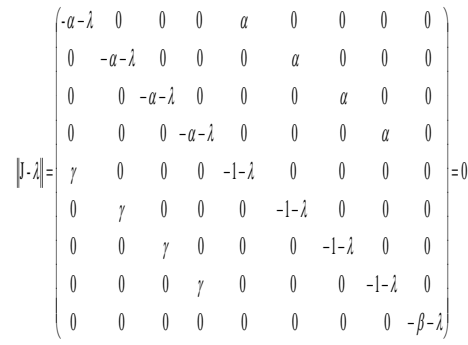

In a next upcoming paper, the proposed system will be analyzed in some more details with its application to the secure communication system and numerical examples.

\section{Weighted-Complex network model}

In the present paper, a weighted-complex dynamic model suggested by [xxx] will be applied for the present dynamic model developed by the authors. The model suggested the following:

$$
\begin{aligned}
& \frac{\partial \mathrm{y}_{\mathrm{j}}}{\partial \mathrm{t}}=\mathrm{g}\left(\mathrm{y}_{\mathrm{j}}\right)+\sum_{j=1}^{M} C_{i j} \Delta \mathrm{y}_{\mathrm{j}}, \\
& 1 \leq i \leq M \\
& \& \\
& \mathrm{y}_{\mathrm{j}}=\left(\mathrm{y}_{\mathrm{i} 1}, \mathrm{y}_{\mathrm{i} 2}, \mathrm{y}_{\mathrm{i} 3} \ldots . ., \mathrm{y}_{\mathrm{im}}\right)^{T} \in \mathrm{R}^{\mathrm{n}}
\end{aligned}
$$

\section{The adaptive-control method}

In this section, the topology-identification is controlled through the following: 
By making use of the following assumption,

$$
\begin{aligned}
& \frac{\partial \hat{\mathrm{y}}_{\mathrm{j}}}{\partial \mathrm{t}}=\mathrm{g}\left(\hat{\mathrm{y}}_{\mathrm{j}}\right)+\sum_{j=1}^{M} \hat{\mathrm{C}}_{i j} \Delta \hat{\mathrm{y}}_{\mathrm{j}}+\zeta_{i} \\
& 1 \leq i \leq M \\
& \& \\
& \hat{\mathrm{y}}_{\mathrm{j}}=\left(\hat{\mathrm{y}}_{\mathrm{i} 1}, \hat{\mathrm{y}}_{\mathrm{i} 2}, \hat{\mathrm{y}}_{\mathrm{i} 3} \ldots . ., \hat{\mathrm{y}}_{\mathrm{im}}\right)^{T} \in \mathrm{R}^{\mathrm{n}}
\end{aligned}
$$

$$
\begin{aligned}
& \dot{\bar{y}}=\hat{\mathrm{y}}_{\mathrm{j}}-\mathrm{y}_{\mathrm{j}} \\
& \& \\
& \bar{C}=\hat{\mathrm{C}}_{\mathrm{j}}-\mathrm{C}_{\mathrm{j}}
\end{aligned}
$$

Then the error system formula will take the following form:

$$
\begin{aligned}
& \dot{\bar{y}}=\mathrm{g}\left(\hat{y}_{\mathrm{j}}\right)-\mathrm{g}\left(\mathrm{y}_{\mathrm{j}}\right)+\sum_{j=1}^{M} \bar{C}_{i j} \Delta \mathrm{y}_{\mathrm{j}}+\sum_{j=1}^{M} C_{i j} \bar{y}_{\mathrm{g}}+\zeta_{i} \\
& \text { With } \\
& \bar{y}=\hat{\mathrm{y}}_{\mathrm{j}}-\mathrm{y}_{\mathrm{j}} \\
& \bar{C}=\hat{\mathrm{C}}_{\mathrm{j}}-\mathrm{C}_{\mathrm{j}}
\end{aligned}
$$

\section{Numerical results and discussion}

\subsection{Test example (1)}

In the present paper, the proposed system will be analyzed for the following numerical data:

$$
\alpha=36, \gamma=14.5, \beta=3
$$

The analysis herein, will contain the analysis of the Equilibrium, by solving eq. (28), and the corresponding results are shown in table (1).

Table 1. Equilibrium of the new system obtained by equation (11)

\begin{tabular}{|c|c|c|c|c|c|c|c|c|c|}
\hline & $u_{1}$ & $u_{2}$ & $u_{3}$ & $u_{4}$ & $u_{5}$ & $u_{6}$ & $u_{7}$ & $u_{8}$ & $u_{9}$ \\
\hline$e_{1}$ & 0 & 0 & 0 & 0 & 0 & 0 & 0 & 0 & 13.5 \\
\hline$e_{2}$ & -6.6 & -6.6 & 14.5 & -6.6 & -6.6 & -6.6 & 14.5 & -6.6 & 13.6 \\
\hline$e_{3}$ & 6.6 & 6.6 & 14.6 & 6.6 & 6.6 & 6.6 & 14.6 & 6.6 & 13.6 \\
\hline
\end{tabular}

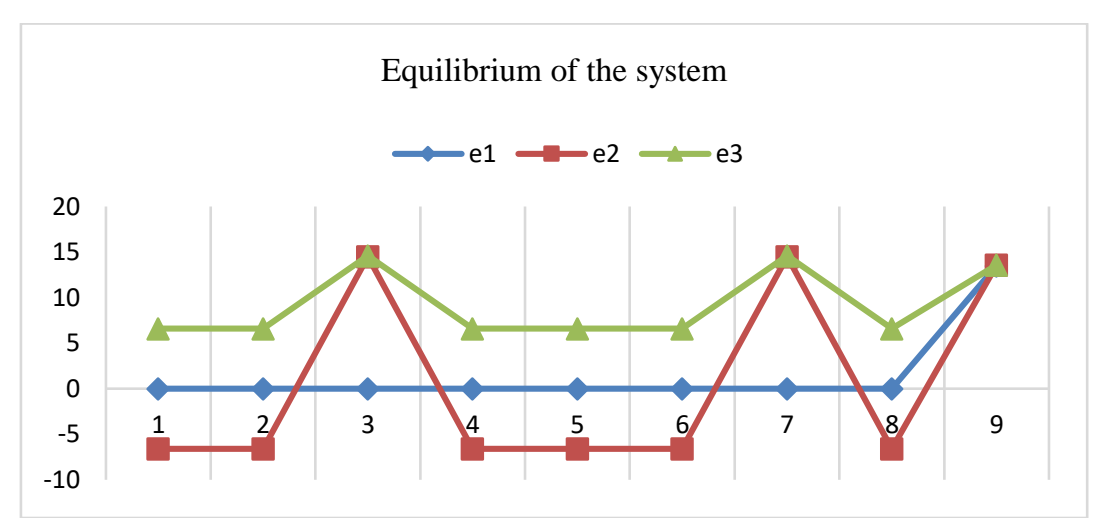

Figure 1. Equilibrium of the new system 
Following up the numerical analysis, by computing the eigenvalues for the obtained new system under the prescribed numerical parameters and the initial condition. The results are shown in table (2). The Fourth order Runge-Kutta method is used in the computation and by making use of the pre-last equation in the system given by equation (28), the values of the $u_{9}$ are obtained.

Table 2. Eigenvalues of the new system obtained by equation (11)

\begin{tabular}{l|l|l|l|l|l|l|l|l|l}
\hline & $\lambda_{1}$ & $\lambda_{2}$ & $\lambda_{3}$ & $\lambda_{4}$ & $\lambda_{5}$ & $\lambda_{6}$ & $\lambda_{7}$ & $\lambda_{8}$ & $\lambda_{9}$ \\
\hline$e_{1}$ & 36 & 14.5 & -2.99 & -35.99 & 14.5 & -2.99 & 0 & 0 & 0 \\
\hline$e_{2}$ & -5.19 & -5.19 & 0.09 & 0.09 & -42.75 & 6.09 & 0 & 0 & 0 \\
\hline$e_{3}$ & -25 & -25 & 0.319 & 0.319 & 0.319 & 0.319 & 0 & 0 & 0 \\
\hline
\end{tabular}

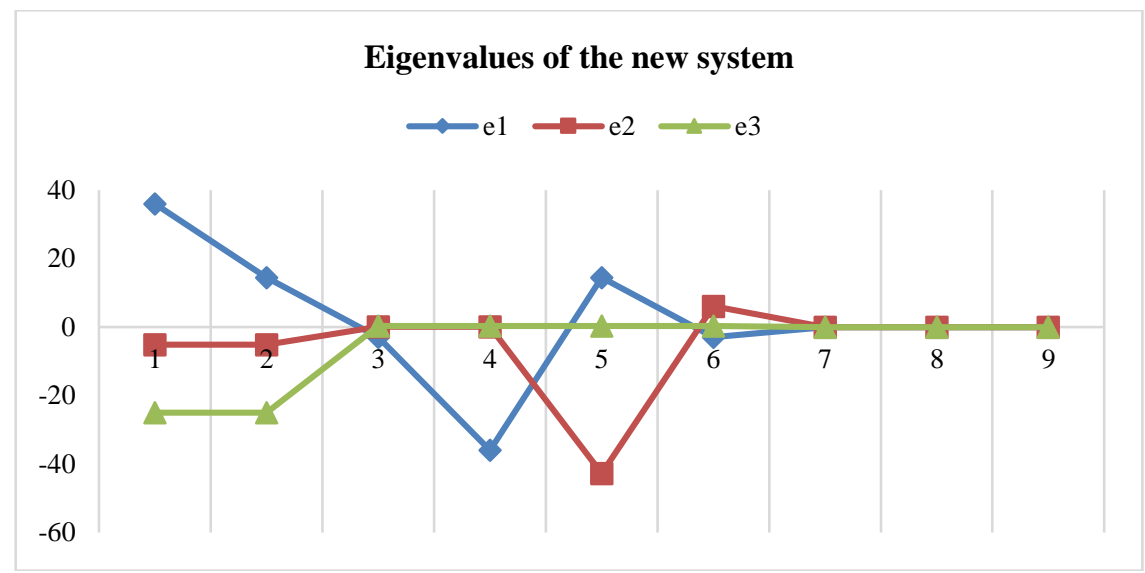

Figure 2. Eigenvalues of the new system

\subsection{Test example (2)}

A simple example is just to clarify the suitability of the adaptive controlling model when applied to the dynamic model developed by the authors. It is well known that the Lorenz chaotic system is known as:

$$
\dot{\mathrm{y}}=\left[\begin{array}{ccc}
-\xi & \xi & 0 \\
\zeta & -1 & 0 \\
0 & 0 & -\varpi
\end{array}\right]\left[\begin{array}{l}
\mathrm{y}_{1} \\
\mathrm{y}_{2} \\
\mathrm{y}_{3}
\end{array}\right]+\left[\begin{array}{l}
0 \\
-\mathrm{y}_{1} \mathrm{y}_{3} \\
\mathrm{y}_{1} \mathrm{y}_{2}
\end{array}\right]
$$

Putting

$\xi=10, \varpi=1.5, \zeta=28$

By considering the complex dynamic network consists of four nodes as shown in figure (3).

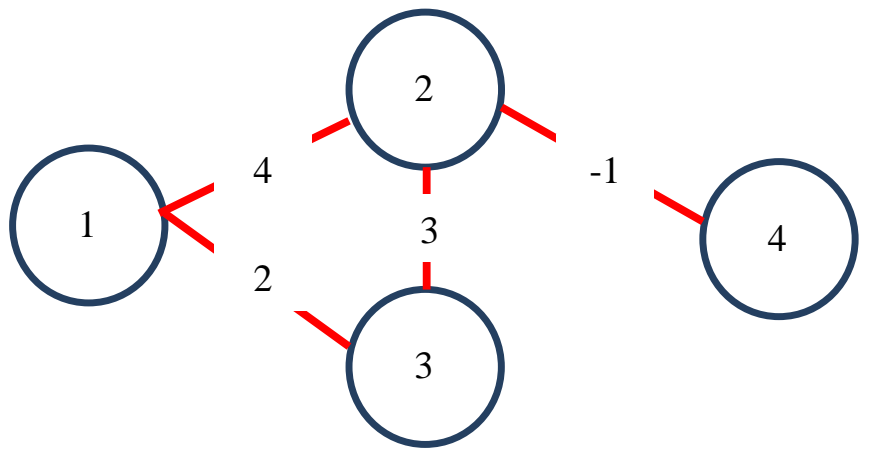

Figure 3. Topology model 


\subsection{Estimation of the topology model}

In this section, we solved a simple example, to study the effectiveness adaptive control method on the system developed. The numerical results are shown both in figure (4) and table (3). The results gave a good approximation and well representation.

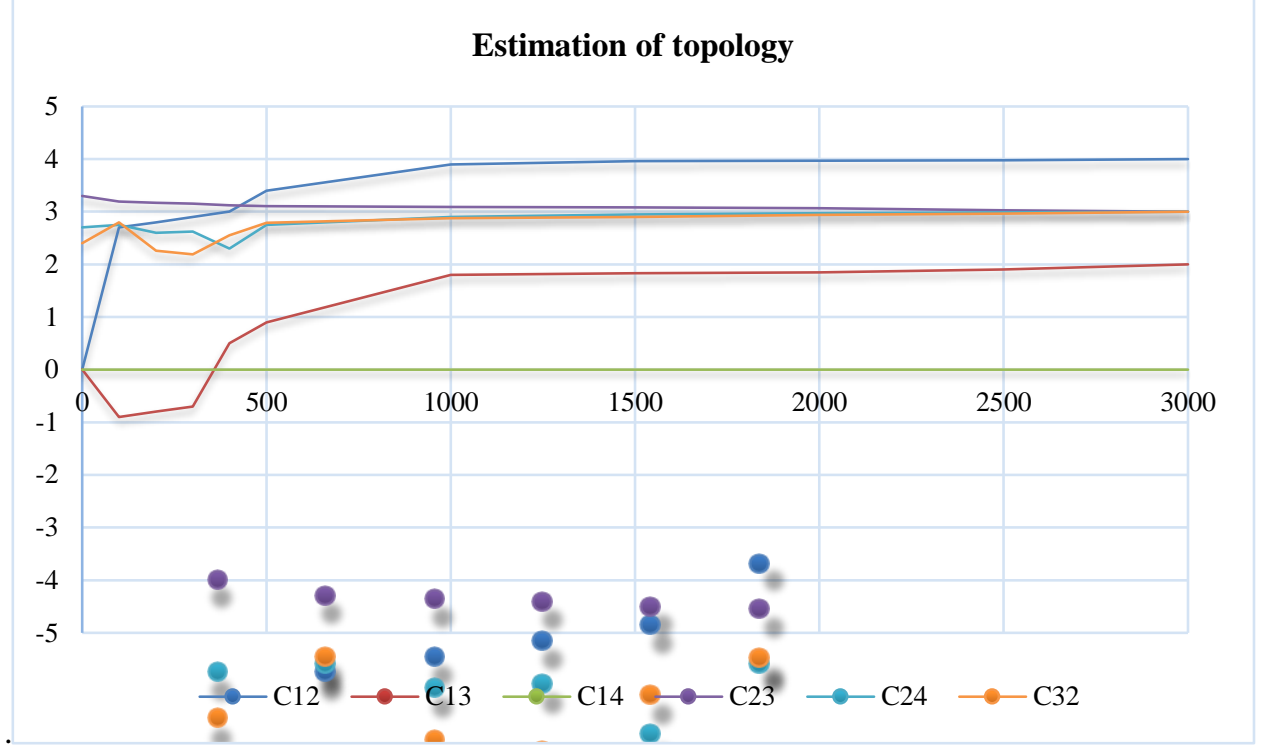

Figure 4. The estimation of the topology model

Table 3. Numerical Estimation of the topology model

\begin{tabular}{|c|c|c|c|c|c|c|}
\hline Time & $\mathrm{C} 12$ & $\mathrm{C} 13$ & $\mathrm{C} 14$ & $\mathrm{C} 23$ & $\mathrm{C} 24$ & $\mathrm{C} 32$ \\
\hline 0 & 0 & 0 & 0 & 3.3 & 2.7 & 2.4 \\
\hline 100 & 2.7 & -0.9 & 0 & 3.19 & 2.75 & 2.8 \\
\hline 200 & 2.8 & -0.8 & 0 & 3.17 & 2.6 & 2.26 \\
\hline 300 & 2.9 & -0.7 & 0 & 3.15 & 2.62 & 2.19 \\
\hline 400 & 3 & 0.5 & 0 & 3.12 & 2.3 & 2.55 \\
\hline 500 & 3.4 & 0.9 & 0 & 3.11 & 2.75 & 2.79 \\
\hline 1000 & 3.9 & 1.8 & 0 & 3.09 & 2.9 & 2.88 \\
\hline 1500 & 3.96 & 1.83 & 0 & 3.08 & 2.95 & 2.9 \\
\hline 2000 & 3.97 & 1.85 & 0 & 3.07 & 2.97 & 2.94 \\
\hline 2500 & 3.98 & 1.9 & 0 & 3.03 & 2.99 & 2.96 \\
\hline 3000 & 4 & 2 & 0 & 3 & 3 & 3 \\
\hline
\end{tabular}

\section{Conclusions}

The present paper studied the geometry which plays an important role in the analysis of dynamic systems. The paper used a new dynamic model developed by the author in a previous paper. The adaptive control method is applied herein, and the results gave a good approximation and presentation for the proposed dynamic system.

\section{References}

[1] E.N Lorenz, "Deterministic non-periodic flow", J Atmos Sci, Vol. 20, 1963, pp130-41.

[2] A. C. ,Fowler, M.J. McGuiness, J.D. Gibbon. "The complex Lorenz equations". Phys D Vol. 4, 1982, pp 13963.

[3] A. Rauh, L.Hannibal, N. Abraham. "Global stability properties of the complex Lorenz model". Phys D, Vol. 99,1996, pp 45-58.

[4] S. G. Ahmed and Mishref, S. A., "A new alternative approach to free-moving boundary problems", Eight International Conference for Free and Moving Boundary Problems, September, 2005, Spain 
[5] E.E. Mahmoud, "Generation and suppression of a new hyper chaotic nonlinear model with complex variables". Appl Math Model, Vol. 38, 2014, pp 4445-59.

[6] J. Gibbon and M. Mc Guinness, "The real and complex Lorenz equations in rotating fluids and lasers", Phys D, Vol. 5(1), 1982, pp108-122.

[7] X. Gómez-Mont, Flores-Godoy, J. J., Fernández-Anaya, "Some attractors in the extended complex Lorenz model", Int. J. Bifurcat. Chaos, Vol Vol. 23 (9), 2013, pp 1-15

[8] P. Reiterer, C. Lainscsek, F. Schürrer, C. Letellier and J. Maquet, "Anine-dimensional Lorenz system to study high-dimensional chaos", J. Phys. A, Math. Gen., Vol. 31 (34), 1998, pp 7121-7139.

[9] P. C. Rech, "Delimiting hyper chaotic regions in parameter planes of a 5D continuous-time dynamical system", Appl. Math. Comput. Vol. 247, 2014, pp 13-17.

[10] W. Wen-Juan, C. Zeng-Qiang and Y. Zhu-Zhi, "Local bifurcation analysis of a four-dimensional hyper chaotic system", Chin. Phys. B, Vol. 17 (7), 2008, pp 2420-2432.

[11]Y. Chen and Q. Yang, "Dynamics of a hyper chaotic Lorenz-type system", Nonlinear Dyn. Vol. 77(3), 2014, pp569-581.

[12]M. J. Correira and P. C. Rech, "Hyper chaotic states in the parameter space, Applied Mathematics and Computation, Vol. 218 (12), 2012, pp6711-6715

[13] B. Bao, J. Xu, Z. Liu and Z. Ma, "Hyper chaos from an augmented Lüsystem", Int. J. Bifurcation Chaos Vol. 20(11), 2010, pp3689-3698

[14]N. Smaoui, M. Zribi, and T. Elmokadem, "A novel secure communication scheme based on the KarhunenLo'eve decomposition and the synchronization of hyper chaotic L"u systems", Nonlinear Dynamics Vol. 90, 2017, pp 271-285.

[15]C. Sun and Q. Xu, Generating chaos from two three-dimensional rigorous linear systems via a novel switching control approach, International Journal of Bifurcation and Chaos, Vol. 26, 2016, pp 1-11

[16]L. Chen, Y. Chai, and R. Wu, "Linear matrix inequality criteria for robust synchronization of uncertain fractional-order chaotic systems," Chaos, An Interdisciplinary Journal of Nonlinear Science Vol. 21, 2011, pp 043-107

[17]L. A. Q. T'ellez, S. C. Moreno, O. R. Jaimes, J. J. F. Godoy, and G. F. Anaya, "Dynamic analysis of a L"u model in six dimensions and its projections," International Journal of Nonlinear Sciences and Numerical, Vol. $18,2017,371-384$.

[18]N. Wang, J. Wang, "Fluctuation model for stock market index based on continuous percolation", $J$. Beijingjiaotong University, Vol. 28 (6), 2004, pp 36-38.

[19] Y. Fang, T.G. Kincaid, "Stability analysis of dynamical neural networks", IEEE Trans. Neural Networks, Vol. 7 (4) ,1996, pp 996-1006.

[20]D.J. Watts, S.H. Strogatz, "Collective dynamics of 'small-world' networks", Nature 391 (4) ,1998, pp440-442.

[21] A.-L. Barabasi, R. Albet, "Emergence of scaling in random networks”, Science Vol. 286 (15) ,1999, pp 509-512.

[22] L.M. Pecora, T.L. Carroll, "Master stability function for synchronized coupled systems", Phys. Rev. Lett. Vol. 80 (10), 1998, pp 2109-2112.

[23]X.F. Wang, G. Chen, "Synchronization in small-world dynamical networks", Int. J. Bifur. Chaos Vol. 12 (1) 2002, pp 187-192.

[24]X.F. Wang, G. Chen, "Synchronization in scale free dynamical networks, robustness and fragility", IEEE Trans. Circuits Syst. I, Vol. 49 (1), 2002, pp 54-62.

[25]I.V. Belykh, V.N. Belykh, M. Hasler, "Connection graph stability method for synchronized coupled chaotic systems", Physica D, Vol. 195, 2004, pp 159-187.

[26] J. Lï,X. Yu, G. Chen, "Chaos synchronization of general complex dynamical networks", Physica A, Vol. 334 (1-2), 2004, pp 281-302.

[27]J. Lï; G. Chen, "A time-varying complex dynamical network models and its controlled synchronization criteria", IEEE Trans. Automatic Control Vol. 50 (6), 2005, pp 841-846.

[28] J. Zhou, J. Lu, J. Lü,"Adaptive synchronization of an uncertain complex dynamical network", IEEE Trans. Automatic Control, Vol. 51 (4), 2006, pp 652-656.

[29] W.K.S. Tang, Y. Mao, L. Kocarev, "Identification and monitoring of neural network", IEEE International Symposium on Circuits and Systems, 27-30, May 2007, p p 2646-2649.

[30] S. Goto, T. Nishioka, M. Kanehisa, "Chemical database for enzyme reactions", Bioinformatics, Vol. 14 (7) ,1998, pp 591-599.

[31] A.B. Horne, T.C. Hodgman, H.D. Spence, A.R. Dalby, "Constructing an enzyme-centric view of metabolism", Bioinformatics, Vol. 20 (13), 2004, pp 2050-2055. 
[32] C.W. Jin, I. Marsden, X.Q. Chen, X.B. Liao, "Dynamic DNA contacts observed in the NMR structure of winged helix protein-DNA complex", J. Mol. Biol. Vol. 289, 1999 p p 683-690

[33] M. Lellis Thivagar, Abdulsattar Abdullah Hamad, "A theoretical implementation for a Proposed HyperComplex Chaotic System", J. of Intelligent and Fuzzy Systems, 2019 (Accepted for publication).

[34] M.Lellis Thivagar, \& C. Richard, "On Nano forms of weakly open sets". International Journal Mathematics and Statistics Invention, Vol. 1, 2013, pp 31-37.

[35] M.Lellis Thivagar, \& C. Richard, "on Nano continuity in a strong form". International Journal of Pure and Applied Mathematics, Vol. 101, 2015, pp 893-904.

[36] M. Lellis Thivagar, V. Sutha Devi, "Computing technique for recruitment process via Nano topology", Sohag Journal of Mathematics, Vol. 3, 2016, pp 37-45.

[37]Benjamin Durakovic, "Design for Additive Manufacturing, Benefits, Trends and Challenges", Periodicals of Engineering and Natural Sciences, Vol.6, (2), 2018, pp.179-191

[38]Benjamin Durakovic, et al, "Lean Manufacturing, Trends and Implementation Issues", Periodical of Engineering and Natural Sciences, Vol. 6, (1), 2018, pp. 130-143

[39] Ramo Palalic, Benjamin Durakovic, "Does Transformational Leadership Matters in Gazelles and Mice, Evidence from Bosnia and Herzegovina?", International Journal of Entrepreneurship and Small Business, Vol. 34, (3), 2018, pp 289-308. 